\title{
L'ELUSIONE COME CATEGORIA AUTONOMA DEL DIRITTO TRIBUTARIO
}

1. L'elusione come categoria autonoma del diritto positivo connotata dall'irreprensibilità del regime formale e dall'intrinseca violazione del precetto normativo. - Il fenomeno dell'elusione può essere ricondotto alla morfologia delle categorie giuridiche trasversali. Infatti, la fattispecie elusiva è una figura diffusa nel sistema normativo, che trova allogazione in vari settori del diritto positivo: il diritto civile; il diritto amministrativo; il diritto tributario. In quest'ultimo comparto, la fenomenologia è conosciuta con il sintagma «elusione fiscale» ${ }^{1}$.

A differenza di altre figure, l'elusione ${ }^{2}$ non costituisce una categoria formale, in quanto manca di una nozione normativa: si tratta, in realtà, di una categoria logica, idonea ad individuare le ipotesi di aggiramento delle norme tributarie. Difatti, eludere una disposizione fiscale significa aggirarla, o mediante il perfezionamento di apposite fattispecie civilistiche, o attraverso comportamenti posti in essere allo scopo di azzerare o di rendere meno gravoso il carico fiscale ${ }^{3}$.

In altri termini, l'elusione si realizza allorquando i mezzi utilizzati per costruire la fattispecie concreta risultano irreprensibili sul piano formale del diritto positivo; diversamente, si rientrerebbe nella figura dell'evasione fiscale.

Sull'elusione tributaria, cfr. S. CIPOLLINA, Elusione fiscale (ad vocem), in Dig. Disc. Priv., Sez. Comm., vol. V Torino, 1990, pagg. 220 e segg.; A. LOVISOLO, Evasione ed elusione tributaria (ad vocem), in Enc. Giur. Treccani, vol. XIII, Roma, 1989, pagg. 1 e segg.; TABELLINI, Elusione fiscale (ad vocem), in Enc. Dir., Aggiornamento, vol. III, Milano, 1999, pagg. 545 e segg.; R. LUPI - G. SEPIO, Elusione fiscale (ad vocem), in II Diritto. Enciclopedia Giuridica del Sole 24-Ore, vol. 6, Milano, 2007, pagg. 1 e segg.; P.M. TABELLINI, L'elusione fiscale, Milano, 1989, passim; TABELLINI, Libertà negoziale ed elusione di imposta, Padova, 1995, passim; KRUSE, II risparmio d'imposta, l'elusione fiscale e l'evasione, in Trattato di diritto tributario, diretto da AMATUCCI, III, Padova, 1994, pagg. 207 e segg.; P. TABELLINI, Elusione tributaria della norma tributaria, Milano, 2007, passim; FIORENTINO, L'Elusione tributaria, Napoli, 1996, passim. Del fenomeno si è occupato il recente Convegno di Studi "Evasione ed elusione fiscale. L'evoluzione del diritto penale tributario a dieci anni dalla riforma del 2000", Taranto 18 ottobre 2010, Auditorium Avv. F. Miro - Tribunale di Taranto.

2 II fenomeno dell'elusione si ricollega alle problematiche civilistiche concernenti il negozio indiretto. Sul negozio indiretto, cfr. DI PAOLO, Negozio indiretto (ad vocem), in Dig. Disc. Priv., Sez. civ., vol. XII, Torino, 1995, pagg. 124 e segg.; SCALFI, Negozio indiretto (ad vocem), in Enc. Giur. Treccani, vol. XX, Roma, 1990, pagg. 1 e segg.

3 In tal senso, cfr. S CIPOLLINA, Elusione fiscale (ad vocem), cit., pagg. 220-221. 
Il procedimento elusivo non è mai occulto ${ }^{4}$, ma caratterizzato da una legittimità formale, considerato che l'obiettivo perseguito dall'elusore è quello di ridurre o di evitare l'onere tributario correlato ad una data operazione economica, senza porre in essere una violazione esplicita del dato normativo: l'intento è di aggirare la fattispecie astratta, per distogliere la norma elusa dal suo naturale àmbito di applicazione.

L'elusione, dunque, appare espressione di una habilité delle parti, di una loro malitia, che, tuttavia, in apparenza, resta nei confini del gioco leale 5 . Inoltre, dato che il procedimento elusivo è caratterizzato da una legittimità formale e da un'intrinseca violazione della ratio legis, esso è connotato dalla manipolazione mediata della fattispecie: l'amministrazione finanziaria, analizzando la fattispecie, decide se, nel singolo caso, si sia verificata un'elusione e se la norma elusa debba essere in concreto applicata.

2. Le origini storiche del fenomeno elusivo: l'agere in fraudem legis. - La fattispecie elusiva presenta origini storiche remote ${ }^{6}$ : già nelle fonti del diritto romano è possibile annoverare tracce dell'istituto. Difatti, l'elusione può essere ricondotta all'antica figura dell'agere in fraudem legis ${ }^{7}$, quale comportamento umano che, sebbene caratterizzato dal rispetto di singole disposizioni, trasgrediva i principi di un settore normativo o quelli desumibili dal complesso ordinamentale.

Alcuni frammenti di Paolo e di Ulpiano, confluiti nella compilazione giustinianea, riassumono i termini della questione, laddove inquadrano l'elusione nella fattispecie del comportamento in frode alla legge, che integra un aggiramento dei principi fondamentali dell'ordinamento giuridico. Secondo l'insegnamento di Paolo, l'agere in fraudem legis, cui la dottrina tributaria riconduce l'elusione fiscale ${ }^{8}$, è il fenomeno per il quale l'agente «contra legem facit qui id facit quod lex prohibet, in fraudem vero qui salvis legis verbis substantiam eius circumvenit»».

La frode alla legge, così delineata, costituisce una sorta di clausola generale, che si adegua alle esigenze dei vari settori dell'ordinamento e, quindi, anche del diritto tributario.

\footnotetext{
Così, cfr. S. CIPOLLINA, Elusione fiscale (ad vocem), cit., pag. 221.

Per tale considerazione, cfr. S. CIPOLLINA, Elusione fiscale (ad vocem), cit., pag. 221.

Un inquadramento storico al fenomeno dell'elusione viene dato da LUPI - G. SEPIO, Elusione fiscale (ad vocem), cit. pagg. 1 e 2 .

\|l tal senso, cfr. R. LUPI - G. SEPIO, Elusione fiscale (ad vocem), cit., pag. 3.

In tal senso, cfr. S. CIPOLLINA, Elusione fiscale (ad vocem), cit., pag. 223.

In particolare, notano giustamente R. LUPI - G. SEPIO, Elusione fiscale (ad vocem), cit., pag. 1, nt. 2, che «già a quell'epoca erano ravvisabili dei casi in cui, in conseguenza delle imperfezioni legislative, i privati riuscivano a porre in essere comunque espedienti elusivi, di fronte ai quali la giurisprudenza non aveva argomenti interpretativi sufficientemente forti per poterli contrastare. Le fonti riportano l'esempio delle numerose leggi, fino alla lex sempronia del 193 a.c., emanate al solo fine di contrastare la diffusa pratica dell'usura, in un contesto in cui i privati riuscivano di volta in volta regolarmente a trovare il metodo per sottrarsi ai divieti imposti dalle leggi».
} 
Nel vigente codice civile, la norma di riferimento è contenuta nell'art. 1344, che disciplina il contratto in frode alla legge, il quale costituisce il mezzo per eludere l'applicazione di una norma imperativa.

Nell'attualità, quindi, il lemma «elusione» individua situazioni nelle quali, malgrado il rispetto della lettera della disposizione, è violata la ratio legis, ossia lo spirito che la norma esprime, con conseguente lesione dell'interesse tutelato ${ }^{10}$.

3. Elusione ed altre figure normative: l'evasione, la simulazione, la frode alla legge e l'abuso del diritto. L'assimilazione del fenomeno elusivo alla frode alla legge. - In realtà, la fattispecie elusiva ${ }^{11}$, pur derivando storicamente dal negozio in frode alla legge, presenta elementi di differenziazione ${ }^{12}$ dagli istituti dell'evasione ${ }^{13}$, della simulazione ${ }^{14}$ e dell'abuso del diritto ${ }^{15}$.

Difatti, l'evasione si ravvisa allorquando il contribuente occulta proventi o deduce costi inesistenti ${ }^{16}$; la simulazione integra ipotesi nelle quali le parti pongono in essere un regolamento soltanto apparente della fattispecie, mentre gli interessi funzionali al negozio sono in concreto inesistenti (simulazione assoluta) o diversi da quelli effettivamente perseguiti (simulazione relativa) ${ }^{17}$.

In altri termini, nella simulazione, le parti fingono, in tutto o in parte, di porre in essere un determinato regolamento contrattuale. Nella fattispecie simulata, quindi, si

In senso conforme, R. LUPI - G. SEPIO, Elusione fiscale (ad vocem), cit., pag. 3.

11 Il fenomeno dell'elusione tributaria è stato $d$ a sempre oggetto di attenzione in dottrina, in quanto ha prospettato una serie di problematiche applicative ed interpretative. Per più approfondite considerazioni, si veda GANGEMI, Alcune note in tema di elusione in campo tributario, in Riv. Crit. Dir. Priv., 1985, pagg. 537 e segg.; G. TREMONTI, Autonomia contrattuale e normativa tributaria: il problema dell'elusione tributaria, in Riv. Crit. Dir. Priv., 1985, pagg. 511; G. TREMONTI, Elusione figlia della legge, in II Sole 24-Ore del 23 aprile 1986, pag. 18; G. TREMONTI, Autonomia contrattuale e normativa tributaria: il problema dell'elusione tributaria, in Riv. Dir. Fin., 1986, I, pagg. 369 e segg.; S. CIPOLLINA, Elusione fiscale, in Riv. Dir. Fin., 1988, I, pagg. 122 e segg.; P. PACITTO, Attività negoziale, evasione ed elusione: spunti problematici, in Riv. Dir. Fin., 1987, I, pagg. 726; R. LUPI, L'elusione come strumentalizzazione delle regole fiscali, in Rass. Trib., 1994, pagg. 225 e segg.; R. LUPI, Elusione fiscale: modifiche normative e prime sviste interpretative, in Rass. Trib., 1995, pagg. 409 e segg. Un esempio concreto delle interferenze esistenti tra l'elusione, la simulazione e la frode alla legge viene offerto da STEVENATO, Donazione e successiva vendita del terreno edificabile, tra elusione, ipotesi simulatorie e frode alla legge, in Dialoghi di diritto tributario, 2006, pag. 1463. Le differenze tra l'istituto dell'evasione e quello dell'elusione tributaria vengono approfondite in maniera attenta da KRUSE, II risparmio d'imposta, l'elusione fiscale e l'evasione, cit., pagg. 207 e segg.

14 Autorevole e risalente dottrina (HELSEN, Diritto tributario, Milano, 1956, pag. 149) metteva in luce le profonde differenze esistenti tra il negozio simulato e l'elusione tributaria, affermando che «l'elusione dell'imposta sta in netta opposizione col negozio simulato e col negozio dissimulato, i quali altrettanto spesso quanto erroneamente vengono richiamati per combattere l'elusione d'imposta; appunto l'intenzione di evitare l'imposta nei casi genuini di elusione parla sempre in favore della serietà del negozio; questo, non il negozio normale posto dal legislatore come fattispecie, vogliono effettivamente le parti».

15 Per le differenze tra elusione fiscale ed abuso del diritto, si rinvia alla pregevole monografia di P. PISTONE, Abuso del diritto ed elusione fiscale, Padova, 1995, passim.

16 In tal senso, cfr. R. LUPI - G. SEPIO, Elusione fiscale (ad vocem), cit., pag. 2

17 Cfr., ancora, R. LUPI - G. SEPIO, Elusione fiscale (ad vocem), cit., pag. 3, che forniscono un'esemplificazione di comportamenti riconducibili alla simulazione. Si pensi, ad esempio, all'uso di un prestanome o al mascheramento di una certa operazione sotto una definizione giuridica alla quale è ricollegato un trattamento fiscale migliore. In tale ipotesi, l'Amministrazione finanziaria può contrastare tale comportamento attribuendo a chi utilizza il prestanome gli effetti delle operazioni compiute da quest'ultimo, sulla base della disposizione normativa di cui all'art. 37 del D.P.R. 600/1973, concernente l'interposizione fittizia di persona in sede di controllo delle dichiarazioni. 
verifica un'antinomia tra ciò che appare nella rete dei rapporti sociali, ed è visibile ai terzi, e ciò che le parti effettivamente intendono attuare.

Dal punto di vista tributario, la simulazione ${ }^{18}$ assume rilievo allorquando è utilizzato un prestanome, che appare formalmente soggetto passivo d'imposta, nella cui sfera giuridica si produrranno gli effetti dell'atto e a carico del quale si applicherà il tributo; oppure nell'ipotesi in cui viene mascherata una certa operazione, attraverso l'attribuzione di una qualificazione giuridica che comporta l'applicazione di un trattamento fiscale meno oneroso.

L'amministrazione finanziaria potrà contrastare il comportamento simulato, imputando al dominus gli effetti fiscali delle operazioni compiute dal prestanome, mediante l'applicazione dell'art. 37 del D.P.R. 29 settembre 1973, n. 600, concernente l'interposizione fittizia di persona in materia di accertamento tributario.

L'evasione fiscale ${ }^{19}$, per converso, può essere inquadrata nel comportamento contra legem tenuto dal contribuente, il quale, pur essendo obbligato al pagamento di un tributo, se ne sottrae in tutto (evasione totale) o in parte (evasione parziale). Nel comportamento mirato all'evasione, l'azione posta in essere dal soggetto passivo d'imposta non è volta a modificare, nei limiti della legalità formale, la struttura della fattispecie tributaria, ma tende a sfuggire illegalmente alle sue conseguenze.

In forza di questa configurazione, l'evasione fiscale integra un inadempimento colpevole della pretesa tributaria, già validamente sorta attraverso la realizzazione della fattispecie, e presuppone un contegno fraudolento e antitributario del contribuente ${ }^{20}$.

Sulla simulazione applicata al diritto tributario, cfr. DOLIA, Simulazione (Dir. Trib.), in Enc. Giur. Treccani, vol. XXVIII, Roma, 1990, pagg. 1 e segg.; S. COVINO, voce Simulazione (Dir. Trib.), in II Diritto. Enciclopedia Giuridica del Sole 24-Ore, vol. 14, Milano, 2007, pagg. 499-500; I. VIGLIOTTI, Simulazione ed elusione d'imposta, in Dir. Prat. Trib., 1983, II, pagg. 665 e segg., che in commento a Cass., 27 luglio 1982, n. 4328, affronta il problema del rapporto tra i due istituti in relazione all'imposta di registro. Peraltro, incidentalmente dell'istituto della simulazione si è occupato anche A. URICCHIO, Commento all'art. 20 T.U.R., in N. D'AMATI, La nuova disciplina dell'imposta di registro, Torino, 1989, pag. 180. Dell'istituto della simulazione in campo tributario, in relazione al tributo di registro, si è occupata anche la giurisprudenza di merito e di legittimità, nonché l'Amministrazione finanziaria. A titolo esemplificativo, cfr. Comm. Trib. Centr., 2 giugno 1982, n. 2943, in Comm. Trib. Centr., 1983, I, pag. 17; Comm. Trib. Centr., 11 ottobre 1984, n. 8765, in Boll. Trib., 1985, pag. 1270; Comm. Trib. Centr., 30 gennaio 1985, in Comm. Trib. Centr., 1985, I, pag. 89; Cass., 13 marzo 1980, n. 1691, in Comm. Trib. Centr., 1980, II, pag. 776; Cass., 12 luglio 1984, n. 4097, in Foro It., 1984, I, c. 2463; Cass, 1 dicembre 1994, n. 10247, in Corr. Trib., 1995, pag. 463; Cass., 12 novembre 1998, n. 11424, in Riv. Giur. Trib., 1999, pag. 756; Ris. Min. Fin., 22 dicembre 1989, n. 461696, in Corr. Trib., 1990, pag. 501.

19 Sulla tematica dell'evasione fiscale, si rinvia a R. LUPI, Evasione fiscale (ad vocem), in II Diritto. Enciclopedia Giuridica del Sole 24-Ore, vol. 6, Milano, 2007, pagg. 275 e segg.; A. LOVISOLO, Evasione ed elusione tributaria (ad vocem), cit., pagg. 5 e segg.; V. VISCO, Evasione fiscale (ad vocem), in Dig. Disc. Priv., Sez. Comm., vol. V, Torino, 1990, pagg. 291 e segg.; E. GONZALEZ GARCIA, La cosiddetta «evasione fiscale», in Riv. Dir. Fin., 1974, I, pagg. 52 e segg.; A. BRANCACCIO, L'evasione delle imposte sul reddito, in Dir. Prat. Trib., 1978, I, pagg. 96 e segg.; A. GIORGETTI, L'evasione tributaria, Torino, 1964, passim; C.F. GROSSO, L'evasione fiscale, Torino, 1980, passim.

20 All'evasione tributaria viene ricollegato il problema della frode fiscale. In tema, si veda F. LEMME, voce Frode fiscale, in Enc. Giur. Treccani, vol. XIV, Roma, 1989, pag. 3. In particolare, sulla frode fiscale, cfr. R. PASELLA, Questioni interpretative in tema di frode fiscale, in Indice pen., 1979, pagg. 173 e segg.; M. CARATOZZOLO, La frode fiscale, in Dir. e prat. Trib., 1983, pagg. 90 e segg.; B. SANTAMARIA, La frode fiscale, Milano, 1987. 
Appare netta la differenza rispetto alla fattispecie elusiva, la quale ha sempre uno sviluppo palese e formalmente legittimo, che comporta l'intrinseco aggiramento dello spirito della legge.

A differenza del comportamento elusivo, l'evasione costituisce un vero e proprio illecito, che può integrare gli estremi di un'infrazione amministrativa, che implica l'applicazione di sanzioni tributarie amministrative, ovvero, nelle ipotesi più gravi, quelli di un reato penale, che comporta l'irrogazione di sanzioni tributarie penali.

Pertanto, va ribadita la netta distinzione tra l'evasione e l'elusione: la prima costituisce un comportamento in violazione diretta di una norma tributaria; la seconda comporta l'apparente osservanza del precetto normativo e la sua violazione implicita, laddove si configura come meccanismo mediante il quale il contribuente mira ad evitare il prelievo tributario, ricorrendo ad apposite «scappatoie» al limite della legalità. Di qui, l'assimilazione del fenomeno elusivo alla frode alla legge, piuttosto che alla violazione diretta del precetto normativo.

4. Le differenze concettuali tra l'elusione, l'evasione e l'erosione. - Malgrado la fondatezza della conclusione teoretica, sul piano applicativo sussistono stretti legami tra l'evasione fiscale, l'elusione tributaria e l'erosione. I tre fenomeni, pur rivestendo caratteristiche differenti, presentano un denominatore comune: la vocazione unitaria alla riduzione del gettito tributario.

Tuttavia, la differenza tra le fattispecie può assumere connotati così labili da rendere difficile la distinzione tra i comportamenti conclamati. Difatti, come si è visto, l'evasione configura un comportamento illegale, che viola una disposizione normativa, in conseguenza del quale il contribuente versa allo Stato meno di quanto dovrebbe o si sottrae all'obbligo tributario.

L'elusione costituisce un quid minus, che si sostanzia nell'utilizzo consapevole delle imperfezioni e delle lacune delle leggi tributarie per ridurre l'onere fiscale, nel rispetto formale della normativa di diritto positivo. La differenza tra l'evasione e l'elusione, quindi, appare netta sul piano formale, sebbene dal punto di vista sostanziale gli obiettivi cui tendono i due comportamenti risultano pressoché identici.

L'erosione, invece, è caratterizzata dalla previsione normativa di deroghe ai principi generali d'imposizione e di trattamenti privilegiati legati al riconoscimento di regimi fiscali agevolati. Nondimeno, anche la distinzione tra l'elusione e l'erosione può assumere connotazioni impercettibili, qualora le agevolazioni siano intenzionalmente utilizzate in maniera più ampia di quanto programmato o supposto dal legislatore. In tale ipotesi, il regime agevolato può costituire fonte di comportamenti elusivi.

Dal punto di vista concreto, dunque, la differenziazione dell'attività elusiva dai fenomeni dell'evasione e dell'erosione può risultare più ardua della mera rappresentazione teoretica. 
5. L'esercizio del diritto soggettivo in difformità dai suoi limiti funzionali: l'elusione fiscale e l'abuso del diritto. L'intento elusivo come artificio soggettivo avente una specifica rilevanza giuridica. - Nella categoria «abuso del diritto» ${ }^{21}$, il diritto soggettivo cessa di ricevere tutela, in quanto esercitato in difformità dai suoi limiti funzionali: il comportamento del titolare del diritto, malgrado la conformità formale alla disposizione normativa, tradisce le finalità intrinseche della norma.

La figura, che presenta notevoli affinità con la fattispecie dell'elusione fiscale ${ }^{22}$, sulla scia della prassi dei paesi di common law, ha avuto di recente un inusuale risvolto applicativo in materia tributaria, per effetto della giurisprudenza di legittimità e comunitaria ${ }^{23}$.

La giurisprudenza della Corte Suprema, che, nell'adattare il principio al settore delle imposte dirette, ne rinveniva le origini nell'ordinamento comunitario, in seguito, ha ricostruito l'abuso del diritto come figura immanente all'ordinamento italiano, avente fondamento nell'art. 53 Cost., che delinea il criterio della capacità contributi$\mathrm{va}^{24} \mathrm{e}$ il parametro della progressività del sistema tributario.

Nella prassi della giurisprudenza tributaria e di legittimità, la figura, in mancanza di una nozione normativa unitaria, è stata applicata alle operazioni dogana-

Per una disamina generale sull'istituto dell'abuso del diritto, cfr. U. NATOLI, Note preliminari ad una teoria dell'abuso del diritto nell'ordinamento italiano, in Riv. Trim., 1958, pagg. 18 e segg.; V. GIORGIANNI, L'abuso del diritto nella teoria della norma giuridica, Milano, 1963; S. ROMANO, voce Abuso del diritto, c) Diritto attuale, in Enc. Dir., vol. I, Milano, 1958, pagg. 166 e segg.; S. PATTI, Abuso del diritto (ad vocem), in II Diritto. Enciclopedia Giuridica del Sole 24-Ore, vol. 1, Milano, 2007, pagg. 1 e segg.; C. SALVI, voce Abuso del diritto I) Diritto civile, in Enc. Giur. Treccani, vol. I, Roma, 1988, pagg. 1 e segg.; M. D'AMELIO, voce Abuso del diritto, in Nov. Dig. It., vol. I, Torino, 1957, pagg. 95-96; A. DI FRANCIA, L'abuso del diritto nella legislazione, nella dottrina e nella giurisprudenza italiana, in Inchieste di diritto comparato, 7, L'abuso del diritto, a cura di M. ROTONDI, Padova, 1979, pagg. 115 e segg.; F. SANTORO PASSARELLI, Dottrine generali del diritto civile, $8^{a}$ ed., Napoli, 1964, pag. 76; G. CATTANEO, Buona fede obiettiva e abuso del diritto, in Riv. Trim. dir. e proc. Civ., 1971, pagg. 613 e segg.; P. RESCIGNO, L'abuso del diritto, in Riv. Dir. Civ., 1965, I, pagg. 205 e segg.; P. RESCIGNO, L'abuso del diritto, Bologna, 1998; M. DOSSETTI, Orientamenti giurisprudenziali in tema di abuso del diritto, in Giur. It., I, 1, pagg. 1573 e segg.; SACCO, L'abuso del diritto soggettivo, in Trattato di diritto civile, diretto da SACCO, Torino, 2001; RESTIVO, Contributo a d una teoria dell'abuso del diritto, Milano, 2007; CASTRONOVO, Abuso del diritto come illecito atipico?, in Europa e Dir. Priv., 2006, pagg. 1051 e segg.; M. MESSINA, L'abuso del diritto, Napoli, 2004; F. GAZZONI, Manuale di diritto privato, XIV ed., Napoli, 2009, pag. 57; P. PERLINGIERI - P. FEMIA, in P. PERLINGIERI Manuale di diritto civile, $6^{a}$ ed., Napoli, 2007, pagg. 80-81; A. TORRENTE - P. SCHLESINGER, Manuale di diritto privato, $19^{\mathrm{a}}$ ed., Milano, 2009, pagg. 77-78.

22 Recentemente l'elusione fiscale è stata estesa anche al controllo sulle società estere. Sul punto, si veda l'articolo di D. AVOLIO - B. SANTACROCE, Sulle controllate estere serve giusto equilibrio, in II Sole 24-Ore del 23 giugno 2010, n. 171 , pag. 33.

23 Si vedano, in particolare, le sentenze della Suprema Corte, che hanno costituito tappe significative per arrivare ad applicare il principio del divieto dell'abuso del diritto an che in materia fiscale. In tal senso, cfr. Cass., 10 maggio 2005, n. 22932; Cass., 12 maggio 2005, n. 20826; Cass., 21 ottobre 2005, n. 20398. In verità, l'apertura della giurisprudenza di legittimità, in recepimento di ciò che stava avvenendo a livello comunitario, costituisce anche una reazione ai comportamenti, sempre più diffusi, posti in essere dai contribuenti per addivenire ad un illecito risparmio di imposta. Difatti, ci si era accorti che, con il passare del tempo, la normativa concernente l'elusione tributaria era insufficiente a contrastare il fenomeno, giacché il risparmio di imposta veniva realizzato attraverso manovre complesse, difficilmente arginabili con le norme sull'elusione e sull'evasione fiscale.

24 Sul principio di capacità contributiva, si rinvia a R. LUPI, voce Capacità contributiva, in II Diritto. Enciclopedia Giuridica del Sole 24-Ore, vol. 2, Milano, 2007, pagg. 683 e segg. 
$\mathrm{li}^{25}$, all'ICI26, all' imposta di registro ${ }^{27}$, alla tassazione delle tipologie contrattuali ${ }^{28}$, alla materia dell' accertamento fiscale ${ }^{29}$, a quella del rimborso delle imposte ${ }^{30}$, ai profili tri-

In tal senso, si veda Cass., 15 settembre 2009, n. 19827. Per le prime osservazioni sulla decisione della Suprema corte, si rinvia a F. FALCONE - A. IORIO, L'abuso del diritto colpisce anche le dogane, in II Sole 24-Ore del 17 settembre 2009 , n. 256 , pag. 35 , che mettono in luce come «l'abuso del diritto colpisce anche le operazioni doganali se per eludere i contingentamenti e le restrizioni sull'importazione di una determinata merce viene utilizzata una società ritenuta prestanome. Ciò perché è immanente nel diritto comunitario, interessato direttamente dai dazi doganali, una clausola generale "antiabusiva" per delegittimare le operazioni commerciali realizzate al fine di ottenere indebite agevolazioni daziarie». Peraltro, la materia dell'abuso del diritto, ricollegata al problema dei dazi doganali, non ha interessato la sola giurisprudenza di legittimità, ma anche quella di merito. A titolo esemplificativo, cfr. Comm. Trib. Reg. Liguria, sent. 18 marzo 2009, la quale ha trattato la tematica in relazione al più vasto problema delle importazioni irregolari.

In particolare, la tematica dell'abuso del diritto ricollegata all'applicazione dell'ICl è stata oggetto della decisione adottata da Cass., 30 novembre 2009, n. 25127, la quale si è occupata del trattamento delle aree pertinenziali, nell'ambito dell'imposta comunale sugli immobili. La Suprema Corte non ha mancato di osservare che il vincolo pertinenziale è opponibile all'Amministrazione finanziaria solo quando è sorretto da esigenze di natura economica, ornamentale o altro. Di conseguenza, non sarà opponibile qualora sia giustificato da mere ragioni di risparmio di imposta. Pertanto, l'impresa edilizia, che simula un rapporto pertinenziale all'esclusivo fine di corrispondere l'imposta comunale sugli immobili in misura ridotta, incorrerà nell'abuso del diritto, categoria giuridica generale desunta dalla giurisprudenza di legittimità dal disposto di cui all'art. 53 Cost. In argomento, si rinvia a L. LOVEC$\mathrm{CHIO}$, Abuso del diritto applicabile alla disciplina Ici, in II Sole 24-Ore del 2 dicembre 2009, n. 332, pag. 33, il quale, fornendo un primo commento alla decisione in esame, sottolinea che «L'abuso del diritto debutta anche nell'Ici. L'impresa che simula un vincolo pertinenziale per evitare il pagamento dell'Ici su un'area edificabile mette in atto un comportamento inopponibile all'ente impositore, in quanto contrario alle regole che presidiano l'applicazione del tributo».

27 Per un'applicazione del principio del divieto dell'abuso del diritto all'imposta di registro, si rinvia alla decisione della Comm. Trib. Prov. Reggio Emilia, del 09/10/2009, in Giur. Trib., 2010, con nota di BEGHINI, L'imposta di registro e l'interpretazione degli atti incentrata sulla sostanza economica nell' «abracadabra» nell'abuso del diritto. In particolare, la decisione ha configurato una fattispecie elusiva riconducibile all'abuso del diritto, ai fini dell'applicazione dell'imposta di registro, nell'operazione di conferimento in società di beni in natura (nel caso di specie, immobili), gravati da debiti, e di successiva cessione delle partecipazioni. In altri termini, secondo la ricostruzione fornita da questa giurisprudenza tributaria di merito, si tratta di un negozio giuridico posto in essere con abuso del diritto. Peraltro, la materia dell'imposta di registro è stata oggetto di attenzione in relazione al problema dell'elusione tributaria. Sul punto, si rinvia, per approfondimenti, allo Studio del CNN (est. G. PETRELLI) n. 95/2003/ $\mathrm{T}$, Imposta di registro. Elusione fiscale. Interpretazione e riqualificazione degli atti, approvato dalla Commissione studi tributari il 26 marzo 2004 e consultabile in CNN Notizie del 4 maggio 2004. Pregevole appare, altresì, la ricostruzione fornita da G. MARONGIU, L'elusione nell'imposta di registro tra l'abuso del diritto e l'abuso del potere, in Dir. Prat. Trib., 2008, pagg. 107 e segg.

In materia, recentemente si è pronunciata anche la Suprema Corte (Cass., 19 maggio 2010, n. 12249), affermando che la presenza di un contratto tipico tra le parti non esclude, in via generale, l'abuso di diritto, quando la tipologia contrattuale prescelta cela la natura abusiva dell'operazione, finalizzata ad ottenere un cospicuo risparmio di imposta. Di conseguenza, l'Amministrazione finanziaria può disconoscere le operazioni (ossia, i contratti conclusi tra le parti) prive di valide ragioni economiche, che mirano ad eludere le leggi fiscali, giacché poste in essere al solo fine di ottenere un indebito risparmio d'imposta. Per un primo commento alla decisione, cfr. F. FALCO$\mathrm{NE}-\mathrm{A}$. IORIO, Abuso del diritto per contratti tipici, in II Sole 24-Ore del 21 maggio 2010, n. 138, pag. 33.

29 In materia, si veda il contributo interpretativo offerto da A. IORIO, Per l'abuso del diritto il limite del beneficio, in II Sole 24-Ore del 3 marzo 2010, n. 61, pag. 36, il quale mette in luce come la recente giurisprudenza di legittimità viene a configurare esistente, anche nell'ordinamento tributario, il divieto dell'abuso del diritto, consistente nel «divieto di conseguire vantaggi fiscali mediante l'uso distorto di strumenti giuridici, pur se non contrastanti con alcuna disposizione normativa, al fine di ottenere vantaggi fiscali in assenza di ragioni economicamente apprezzabili». In particolare, il principio del divieto dell'abuso del diritto, applicato alla tematica dell'accertamento tributario, impone all'Amministrazione finanziaria di dimostrare che il contribuente sottoposto a controllo si sia effettivamente avvantaggiato dall'operazione posta in essere, in violazione del principio predetto. Interessante appare, altresì, la ricostruzione di A. CRISCIONE, Con l'abuso del diritto una spinta ai controlli, in II Sole 24-Ore del 4 marzo 2010, n. 62, pag. 33, il quale sottolinea che "l'abuso del diritto non è più solo un' "invenzione" della Corte di Cassazione, il jolly per le sentenze nei casi in cui ricorrere al tradizionale concetto di elusione offriva appigli troppo deboli. Ora è anche uno strumento usato con una relativa frequenza dagli uffici fiscali, anche se con una certa preferenza per i casi relativi ai grandi contribuenti. $E$ se in qualche regione se ne ammette l'utilizzo come strumento quasi "ordinario" in alcuni casi, in altre l'abuso del diritto è strumento residuale quando le altre forme "tipizzate" di elusione non reggono».

30 In tal senso, cfr. Comm. Trib. Reg. Lombardia, sent. n. 171/28/09, depositata il 23 novembre 2009, la quale ritie- 
butari delle successioni mortis causa ${ }^{31} \mathrm{e}$, da ultimo, si è cercato di estenderla anche al settore delle imposte dirette ${ }^{32}$.

La categoria dell'abuso del diritto, che sembra condividere con l'elusione l'obiettivo di raggirare l'applicazione della norma e di contravvenire alle sue finalità intrinseche, in realtà non può essere assimilata alla fattispecie elusiva, trattandosi di figure tra loro non sovrapponibili.

In questa prospettiva, l'opzione del contribuente per una soluzione idonea a ridurre il gravame impositivo assume connotazione elusiva allorquando si trasforma in un espediente, formalmente lecito, che cagiona la violazione sostanziale del precetto tributario. In altri termini, i comportamenti elusivi costituiscono artifici giuridici, i quali, nell'apparente osservanza della norma, di fatto la violano, perché in contrasto con l'ordine normativo sostanziale.

Pertanto, un elemento essenziale della fattispecie elusiva è costituito dall'artificio soggettivo, che assume specifica rilevanza giuridica. L'intento elusivo, tuttavia, non va inteso come mera consapevolezza dell'autore di porre in essere un atto in contrasto con lo spirito della legge, ma come progetto concreto che informa l'azione e da essa è direttamente rilevato. Dal punto di vista definitorio, quindi, l'elusore è colui che, nel compiere le operazioni programmate, non nasconde la realtà formale, ma raggira lo spirito di una disposizione cogente del sistema normativo.

ne che ci può essere abuso del diritto anche nelle modalità di scelta e nella tempistica della richiesta di rimborso delle imposte, con la conseguente possibilità per l'Amministrazione finanziaria di sindacare anche le procedure scelte dal contribuente. Per un primo commento alla decisione, cfr. A. CRISCIONE - A. IORIO, L'abuso del diritto «invade» i tempi per il rimborso, in ॥ Sole 24-Ore del 28 novembre 2009, n. 328, pag. 25.

31 Pregevole appare in materia la ricostruzione offerta da E. DE MITA, Abuso del diritto inserito a forza nelle successioni, in II Sole 24-Ore, del 13 settembre 2009, n. 252, pag. 25, il quale critica la risoluzione dell'Agenzia delle Entrate del 24 agosto 2009, n. 234/E, che, facendo ricorso al divieto dell'abuso del diritto, ha ritenuto illegittimo il comportamento dell'erede superstite, il quale ha rinunziato, in nome e per conto del chiamato defunto, all'eredità che a quest'ultimo sarebbe spettata. In particolare, l'Amministrazione finanziaria sottolinea che una tale rinunzia, effettuata all'esclusivo scopo di fruire di un risparmio d'imposta, verrebbe a concretizzare l'abuso del diritto. In realtà, l'Autore nota che «nell'imposta di successione la rinuncia all'eredità da parte dell'erede che succede a un chiamato defunto non è automaticamente abuso del diritto. Se c'è rinuncia non c'è tassazione; ma non si può dire che c'è violazione dell'articolo 53 solo perché non c'è tassazione». In altri termini, si mette in luce la pras$\mathrm{si}$, che recentemente si sta diffondendo in seno alla giurisprudenza tributaria (di merito e di legittimità) ed all'Amministrazione finanziaria, di invocare l'abuso del diritto come principio interpretativo. A tal fine, l'autore sottolinea che "l'abuso del diritto non è un principio interpretativo, una specie di "in dubio pro fisco" che vada a scegliere la sola interpretazione favorevole al fisco. Anche se in materia si è fatto riferimento, in una giurisprudenza corriva, all'articolo 53 della Costituzione, non mi pare vi sia una coincidenza fra principio di capacità contributiva e divieto dell'abuso del diritto. L'interpretazione adeguatrice fatta sulla base del principio di capacità contributiva non porta all'identificazione letterale con la nozione di abuso del diritto».

32 In materia, si veda, in particolare, l'ordinanza adottata recentemente dalla Corte di Cassazione il 4 agosto 2010 n. 18055 , con la quale la Suprema Corte sottopone alla Corte Ue il problema della rilevanza in ambito comunitario dell'abuso del diritto nell'imposizione diretta. In altri termini, la Cassazione ha ritenuto opportuno il parere de giudici comunitari in relazione alla rilevanza, rispetto ai principi comunitari, di pratiche di abuso del diritto sulle imposte dirette. Sulla tematica, cfr. A. IORIO - F. FALCONE, Abuso del diritto e imposte dirette al test Corte Ue, in II Sole 24-Ore, del 5 agosto 2010 , n. 213, pag. 21, i quali osservano che «ora risulterà particolarmente importante conoscere l'opinione sul punto dei giudici comunitari sia con riferimento al futuro della nuova definizione, sia, soprattutto, circa il valore che verrà attribuito all'abuso del diritto all'interno dell'imposizione diretta, in ambito comunitario». Peraltro, è opportuno ricordare che della problematica dell'elusione ricollegata alle imposte reddituali si era già occupato in passato A. ZOPPINI, Fattispecie e disciplina dell'elusione nel contesto delle imposte reddituali, in Riv. Dir. Trib., 2002, pagg. 53 e segg. 
Il fenomeno consiste, appunto, nello sfruttamento delle smagliature delle norme tributarie, al fine di realizzare un consistente risparmio d'imposta, alla luce apparente della legalità e nel rispetto formale delle regole.

In questi termini, per aversi una fattispecie elusiva è necessario il concorso di tre requisiti essenziali: l'intento di risparmiare l'imposta; l'anormalità dei procedimenti scelti; l'alleggerimento effettivo del carico fiscale. Il primo elemento include gli altri, i quali assurgono a indici obiettivi della struttura della fattispecie.

6. L'elusione fiscale e il negotium in fraudem fisci. - Per quanto l'elusione fiscale prenda le mosse dal negozio in frode alla legge ${ }^{33}$ (art. 1344 cod. civ.), non pare corretta la riconduzione a questa figura giuridica. Le due fattispecie presentano molteplici elementi discretivi ${ }^{34}$ : l'imperatività della norma tributaria riveste peculiarità diverse dall'imperatività dell'art. 1344 cod. civ., che tende ad impedire che i soggetti dell'ordinamento possano dare surrettizia attuazione a regolamenti negoziali in contrasto con interessi meritevoli di tutela ${ }^{35}$.

In altri termini, l'art. 1344 cod. civ. si riferisce alle norme imperative di diritto privato, le quali, nell'individuare i limiti all'autonomia negoziale36, inibiscono comportamenti diretti a raggirare un'autoregolamentazione dei rapporti privati difforme da quanto astrattamente previsto dal legislatore.

Questa connotazione finalistica esclude che la disposizione possa essere applicata ai negozi in frode al fisco (c.d. negotia in fraudem fisci): l'imperatività dell'art. 1344 cod. civ. non si conforma a tutte le norme inderogabili, ma solo a quelle precettive o proibitive che subordinano il perseguimento di un risultato materiale alla sussistenza di particolari condizioni o dichiarano l'obiettivo programmato direttamente illecito.

In questa tipologia non rientrano le norme tributarie, le quali, pur caratterizzate dal requisito dell'inderogabilità, non considerano determinate situazioni come non meritevoli di protezione o non riconoscono la tutela di altre in presenza di specifiche condizioni, ma assumono talune situazioni della realtà fenomenica come indice di capacità contributiva (ex art. 53 Cost.), al fine di ricollegarvi il tributo ed individuarne il soggetto passivo d'imposta.

Pertanto, nelle norme tributarie viene meno la ratio dell'art. 1344 cod. civ. di colpire pure il risultato, diverso ma analogo a quello previsto dalla norma proibitiva

33 Per i rapporti tra l'elusione e la frode alla legge, cfr. GALLO, Brevi spunti in tema di elusione e frode alla legge (nel reddito d'impresa), in Rass. Trib., 1989, I, pagg. 11 e segg.; GIULIANI, Elusione fiscale, frode alla legge e causa concreta del contratto, in Contratto e impresa, 2007, 2, pag. 455

Sul punto, si rinvia all'approfondita ricostruzione di S. CIPOLLINA, Elusione fiscale (ad vocem), cit., pagg. 222 e segg.

35 In tal senso, cfr. R. LUPI - G. SEPIO, Elusione fiscale (ad vocem), cit., pag. 3

36 Così, in particolare, R. LUPI - G. SEPIO, Elusione fiscale (ad vocem), cit., pagg. 3-4. 
o precettiva, maliziosamente conseguito dalle parti per superare l'ostacolo legislativo alla sua realizzazione ${ }^{37}$.

Inoltre, mentre nel negozio in frode alla legge sussistono sia l'elemento oggettivo e che quello soggettivo, nell'elusione è presente il parametro soggettivo della frode, ma non l'elemento oggettivo della lesione dell'interesse sociale, che l'ordinamento tutela attraverso la previsione del divieto o del precetto. L'oggetto della frode fiscale, quindi, non è ravvisabile nella violazione della norma, ma nella lesione dell'interesse dello Stato alla percezione del tributo.

In realtà, la fraus fisci può essere allogata nella categoria della frode ai creditori, piuttosto che nel negozio in frode alla legge ${ }^{38}$ (ex art. 1344 cod. civ.), considerato che lo Stato, quale soggetto attivo d'imposta, può essere assimilato alla parte attiva del rapporto obbligatorio di diritto privato.

In conclusione, l'elusione fiscale, a differenza del negozio in frode alla legge, può ricevere attuazione non soltanto mediante le tipologie contrattuali e, quindi, abusando delle regole in materia di autonomia privata, ma anche attraverso atti non negoziali: non sono infrequenti le ipotesi in cui il contribuente, titolare di un'impresa di produzione di beni, effettui una manipolazione materiale dei prodotti per beneficiare di un regime fiscale meno gravoso in forza dell'imprecisione della norma tributaria; ovvero le altre ipotesi di chi pone in essere operazioni imponibili al solo scopo di sbloccare i rimborsi dei crediti ${ }^{39}$.

Neppure sono rare le situazioni nelle quali il contribuente, per conseguire un risultato economico, adotta, in luogo della forma contrattuale tipica, una tipologia negoziale anomala o indiretta, mirata a perseguire il medesimo risultato, ma con un evidente risparmio d'imposta: può pensarsi alla situazione in cui, in luogo di una compravendita fiscalmente più onerosa, viene stipulato un mandato a vendere con contestuale rilascio di una procura irrevocabile alla gestione del bene. In questi casi, l'applicazione della norma civilistica (art. 1344 cod. civ.) non permette di arginare il fenomeno dell'elusione fiscale.

Cfr. S. CIPOLLINA, Elusione fiscale (ad vocem), cit., pag. 222.

Per un'approfondita ricostruzione del negozio in frode alla legge, cfr. G. D'AMICO, Libertà di scelta del tipo contrattuale e frode alla legge, Milano, 1992; L. CARRARO, II negozio in frode alla legge, Padova, 1943; G. GIACOBBE, La frode alla legge, Milano, 1968; G. GIACOBBE, voce Frode alla legge, in Enc. Dir., vol. XVIII, Milano, 1969, pagg. 73 e segg.; MORELLO, Frode alla legge, Milano, 1969; G. CRICENTI, I contratti in frode alla legge, Milano, 1996; M. RABITTI, voce Frode alla legge (contratto in), in II Diritto. Enciclopedia Giuridica del Sole 24Ore, vol. 6, Milano, 2007, pagg. 553 e segg.; U. MORELLO, voce Frode alla legge, in Dig. Disc. Priv., Sez. Civ., vol. VIII, Torino, 1992, pagg. 501 e segg.; M. NUZZO, voce Negozio giuridico, IV) Negozio illecito, in Enc. Giur. Treccani, vol. XX, Roma, 1990, pagg. 3 e segg.; F. DI MARZIO, Frode alla legge nei contratti, in Giust. Civ., 1998, II, pagg. 573 e segg.; DI MARZIO, Interposizione reale di persona, simulazione, frode alla legge nei contratti, in Giust. Civ., 2001, II, pag. 433; G. GITTI, II contratto in frode alla legge. Itinerari della giurisprudenza, in Riv. Crit. Dir. Priv., 1989, pagg. 697 e segg.; VELLUZZI, II contratto in frode al terzo: individuazione della fattispecie e rapporti con la frode alla legge, in Rass. Dir. Civ., 2004, pagg. 183 e segg. 
7. Il contegno elusivo e l'interpretazione antielusiva della norma fiscale: la linea di demarcazione tra il comportamento elusivo e il risparmio lecito dell'imposta. La rilevanza della distinzione tra le disposizioni antielusive a struttura ordinaria e la disposizione antielusiva generale. - L'approccio più congruente per la comprensione della figura dell'elusione deve analizzare il rapporto tra il contegno elusivo e l'interpretazione della norma fiscale ${ }^{40}$.

Infatti, l'elusione integra un comportamento del contribuente non contrastabile soltanto per via interpretativa; altrimenti, il raggiro delle disposizioni normative si rileverebbe inidoneo a perseguire lo scopo fraudolento e farebbe fallire il comportamento elusivo. L'attività interpretativa deve svolgersi nel rispetto delle limitazioni imposte dallo spirito e dal sintagma della legge.

In questa prospettiva, l'interpretazione antielusiva può costituire un ostacolo all'elusione, una volta che si sia verificata, ma non appare uno strumento idoneo alla sua inibizione aprioristica ${ }^{41}$.

Per un corretto inquadramento della problematica, occorre individuare la linea di demarcazione tra il comportamento elusivo e il risparmio lecito dell'imposta ${ }^{42}$, ossia tra l'aggiramento malizioso della fattispecie e la scelta fiscalmente meno onerosa per il contribuente. Invero, la linea di confine tra ciò che è lecito e ciò che non lo è non sembra immutabile, ma risente dell'applicazione combinata di due fattori: il grado di evoluzione raggiunto dall'ordinamento giuridico; l'impostazione accolta dall'interprete nell'ermeneutica della norma elusa ${ }^{43}$.

In realtà, le tecniche antielusive presentano non pochi inconvenienti, dato che l'elusione costituisce un fenomeno complesso, che non può essere arginato con strumenti artificiosi, ma richiede interventi legislativi mirati ad introdurre appositi strumenti di repressione ${ }^{44}$.

Il legislatore tributario, nel corso degli anni, ha introdotto una serie di strumenti normativi diretti a neutralizzare i comportamenti elusivi: da un lato, la categoria delle presunzioni legali, al fine individuare con precisione il fatto tassabile; dall'altro, l'abrogazione o la modifica di disposizioni fiscali eccessivamente permissive o contenenti clausole ampie e innominate, tali da prestarsi ad interpretazioni variegate; infine, la previsione di sanzioni tributarie amministrative e penali da applicare ai fenomeni elusivi e non soltanto alle fattispecie di evasione fiscale.

Per un'attenta ricostruzione dell'interpretazione della norma elusa, cfr. S. CIPOLLINA, Elusione fiscale (ad vocem), cit., pagg. 224 e segg.

Cfr. R. LUPI - G. SEPIO, Elusione fiscale (ad vocem), cit., pag. 4

Per tale considerazione, cfr. S. CIPOLLINA, Elusione fiscale (ad vocem), cit., pag. 222.

In tal senso, cfr. S. CIPOLLINA, Elusione fiscale (ad vocem), cit., pag. 222.

Sulla questione degli strumenti volti a reprimere i comportamenti elusivi, cfr. DUS, Categorie ordinanti della fiscalità e norma antielusiva, in Dialoghi, 2007, pagg. 1127 e segg.; BEGHIN, II dividend stripping tra clausole generali antielusive, disposizioni ordinarie con funzione antielusiva e abnorme impiego di strumentazione civilistica, in Riv. Dir. Trib., 2007, II, pagg. 691 e segg. 
Inizialmente, il legislatore italiano ha cercato di porre rimedio all'elusione con interventi settoriali di modifica delle norme in materia di calcolo dell'imposta e di prevenzione dei comportamenti elusivi, senza dettare disposizioni generali sul fenomeno. Ne è derivata una sorta di legislazione dell'emergenza, volta a reprimere specifici comportamenti elusivi, senza alcuna vocazione unificante ${ }^{45}$.

Il protrarsi di questa tecnica legislativa ha determinato una sovrapposizione di criteri normativi, che ha portato all'elaborazione di regole farraginose e contraddittorie. Infatti, per alcuni decenni, il contrasto al fenomeno è stato affidato a disposizioni «rigide», aventi struttura ordinaria e funzione latamente antielusiva, cui è conseguito l'inconveniente di estendere il regime normativo ad ipotesi in cui le preoccupazioni antielusive erano ingiustificate ${ }^{46}$.

Per superare le antinomie prodotte dalle disposizioni a struttura ordinaria, il legislatore ha introdotto nel sistema tributario una norma antielusiva di portata generale (art. 10, legge n. 408/1990), la quale consente all'amministrazione finanziaria di disconoscere il vantaggio conseguito dal contribuente attraverso scappatoie ermeneutiche ed espedienti fattuali ${ }^{47}$.

La norma antielusiva generale è stata inserita nell'art. 37 bis del D.P.R. n. 600/1973 ${ }^{48}$ (Decreto sull'accertamento delle imposte sui redditi), che dispone: «Sono inopponibili all'Amministrazione Finanziaria gli atti, i fatti e i negozi, anche collegati tra loro, privi di valide ragioni economiche, diretti ad aggirare obblighi o divieti previsti dall'ordinamento tributario e ad ottenere riduzioni d'imposta o rimborsi, altrimenti indebiti. L'amministrazione finanziaria disconosce i vantaggi tributari conseguiti mediante gli atti, i fatti e i negozi di cui al 1 comma, applicando le imposte determinate sulla base delle disposizioni eluse, al netto delle imposte dovute per effetto del comportamento inopponibile all'amministrazione».

Il legislatore ha subordinato l'applicazione della disposizione antielusiva ${ }^{49}$ generale a presupposti di natura formale, che conferiscono rilievo alla tassatività delle operazioni elencate (fusioni, scissioni e altre operazioni), e a requisiti di carattere teleologico, che assegnano rilevanza alle finalità che hanno indotto il contribuente ad agire $^{50}$. Infatti, il comportamento del soggetto passivo d'imposta deve essere finaliz-

45 Cfr. R. LUPI - G. SEPIO, Elusione fiscale (ad vocem), cit., pag. 4.

46 Per tali considerazioni, cfr. R. LUPI - G. SEPIO, Elusione fiscale (ad vocem), cit., pag. 4

47 In tal senso, cfr., ancora, R. LUPI - G. SEPIO, Elusione fiscale (ad vocem), cit., pag. 5.

48 Pregevole sul tema appare la ricostruzione fornita da ZIZZO, La nozione di elusione nella clausola generale, in Corr. Trib., 1996, pagg. 3087 e segg., il quale, definendo l'ambito di applicazione della disposizione normativa, rileva che la collocazione della norma «ne condiziona la sfera di applicazione, limitandola a quelle imposte (quelle sui redditi), e a quelle altre il cui accertamento è regolato mediante il rinvio alla normativa in materia di imposte sui redditi».

49 In materia di disposizioni antielusive, cfr. RUSSO, Note in tema di disposizioni antielusive, in Rass. Trib., 1999, pagg. 68 e segg.

50 Cosi R. LUPI - G. SEPIO, Elusione fiscale (ad vocem), cit., pag. 5. 
zato al conseguimento di un indebito vantaggio fiscale e, quindi, deve risultare privo di ragioni economiche legate ad esigenze extrafiscali.

In ogni caso, qualora l'amministrazione finanziaria intenda applicare la disposizione per disconoscere i vantaggi fiscali conseguiti indebitamente dal contribuente ${ }^{51}$, deve chiedere, a pena di nullità, gli opportuni chiarimenti e indicare le motivazioni per le quali ritiene di procedere all'applicazione della disposizione generale antielusiva $^{52}$. La normativa, pertanto, a differenza delle disposizioni antielusive a struttura ordinaria, presenta una portata del tutto diversa, per l'attitudine a conformarsi alla mutevolezza della prassi applicativa.

8. La tutela del contribuente tra interpello ordinario e interpello speciale antielusivo. - Un ulteriore istituto che viene in rilievo nella materia dell'elusione fiscale è costituito dall'interpello speciale antielusivo, strumento a tutela del contribuente, che, per mettersi al riparo da sanzioni fiscali, chiede preventivamente all'amministrazione finanziaria se il comportamento programmato possa essere considerato elusivo ${ }^{53}$.

Questa forma di interpello ${ }^{54}$ ha un ambito di applicazione più ristretto dell'interpello ordinario (art. 11, legge n. 212/2000) (Statuto dei diritti del contribuente ${ }^{55}$ ), in quanto concerne l'applicazione di disposizioni specifiche ${ }^{56}$ : la norma antielusiva generale (art. 37 bis, D.P.R. n. 600/1973); l'interposizione fittizia di persone (art. 37, D.P.R. n. 600/1973); la distinzione tra spese promozionali e spese di rappresentanza; i dividendi esteri; le operazioni infragruppo con non residenti; i conferimenti esteri; la dual income tax.

L'interpello antielusivo consente al contribuente di sollecitare una risposta dell'amministrazione finanziaria e di metterla in mora in caso di omessa risposta. Infatti, in presenza di particolari presupposti, il silenzio dell'amministrazione è equiparato all'accoglimento dell'istanza ${ }^{57}$.

51 Sulle conseguenze derivanti dall'aver posto in essere un comportamento rientrante nella fattispecie elusiva di cui all'art. 37-bis del D.P.R. n. 600/1973, cfr. F. GALLO, Rilevanza penale dell'elusione, in Rass. Trib., 2001, pagg. 327 e segg.; DEL FEDERICO, Elusione e illecito tributario, in Corr. Trib., 2006, pagg. 3113 e segg.

Cfr. R. LUPI - G. SEPIO, Elusione fiscale (ad vocem), cit., pagg. 5-6.

Cfr. R. LUPI - G. SEPIO, Elusione fiscale (ad vocem), cit., pag. 11.

Si tratta di una forma di interpello introdotta nel nostro ordinamento dalla legge n. 413/1991, la quale ha ricevuto attuazione molto più tardi con i decreti ministeriali del 13 giugno 1997 numeri 194 e 195. Per alcune considerazioni su tale tipo di interpello, cfr. ZIZZO, Diritto d'interpello e ruling, in Riv. Dir. Trib., 1992, pagg. 136 e segg.; LA ROSA, Prime considerazioni sul diritto di interpello, in II Fisco, 1992, pagg. 7946 e segg. Per un'attenta ricostruzione dell'istituto dell'interpello, cfr. G. SEPIO, voce Interpello (Dir. Trib.), in II Diritto. Enciclopedia Giuridica del Sole 24-Ore, vol. 8, Milano, 2007, pagg. 116 e segg.

Sullo Statuto del contribuente si rinvia alle attente ricostruzioni di A. URICCHIO, voce Statuto del contribuente, in Digesto Agg., vol. II., Torino, 2003, pagg. 845 e segg.; F. BIANCHI - R. LUPI, voce Statuto del contribuente, in II Diritto. Enciclopedia Giuridica del Sole 24-Ore, vol. 15, Milano, 2007, pagg 419 e segg.; A. FANTOZZI - A. FEDELE (a cura di), Statuto dei diritti del contribuente, Milano, 2005.

56 Per tali considerazioni, si rinvia a R. LUPI - G. SEPIO, Elusione fiscale (ad vocem), cit., pag. 11

57 Così, ancora, R. LUPI - G. SEPIO, Elusione fiscale (ad vocem), cit., pag. 11. 
Invero, le ipotesi più frequenti nelle quali l'interpello speciale antielusivo ha trovato applicazione riguardano proprio la disposizione antielusiva generale, a riprova dell'interesse che l'istituto ha suscitato nella prassi applicativa ${ }^{58}$.

58 Interesse solleva, altresì, lo strumento di cui all'art. 37 bis, comma 8, D.P.R. n. 600/1973, finalizzato alla disapplicazione delle norme antielusive a struttura ordinaria, il quale si pone come alternativa all'interpello speciale antielusivo. La previsione normativa dispone che «le norme tributarie finalizzate a contrastare comportamenti antielusivi possono essere disapplicate qualora il contribuente dimostri che nella particolare fattispecie tali effetti elusivi non potevano verificarsi. A tal fine il contribuente deve presentare istanza al direttore regionale delle entrate competente per territorio, descrivendo compiutamente l'operazione e indicando le disposizioni normative di cui si chiede la disapplicazione». 


\section{L'ELUSIONE COME CATEGORIA AUTONOMA DEL DIRITTO TRIBUTARIO}

Il fenomeno dell'elusione può essere ricondotto alla morfologia delle categorie giuridiche trasversali. Infatti, la fattispecie elusiva è una figura diffusa nel sistema normativo, presente in vari settori del diritto positivo: il diritto civile; il diritto amministrativo; il diritto tributario. In quest'ultimo comparto, la fenomenologia è conosciuta come «elusione fiscale».

A differenza di altre figure, l'elusione non costituisce una categoria formale, in quanto manca di una nozione normativa: si tratta, in realtà, di una categoria logica, idonea ad individuare le ipotesi di aggiramento delle norme tributarie.

Eludere una disposizione fiscale significa aggirarla, o mediante il perfezionamento di apposite fattispecie civilistiche, o attraverso comportamenti posti in essere allo scopo di azzerare o di rendere meno gravoso il carico fiscale.

In altri termini, l'elusione si realizza allorquando i mezzi utilizzati per costruire la fattispecie risultano irreprensibili sul piano formale del diritto positivo; diversamente, si rientrerebbe nella figura dell'evasione fiscale.

La fattispecie elusiva presenta origini storiche remote: già nelle fonti del diritto romano è possibile annoverare tracce dell'istituto. Difatti, l'elusione può essere ricondotta all'antica figura dell'agere in fraudem legis, quale comportamento umano che, sebbene caratterizzato dal rispetto di singole disposizioni, trasgrediva i principi di un settore normativo o quelli desumibili dal complesso ordinamentale.

Nell'attualità, il lemma «elusione» individua situazioni nelle quali, malgrado il rispetto della lettera della disposizione, è violata la ratio legis, ossia lo spirito che la norma esprime, con conseguente lesione dell'interesse tutelato.

In realtà, la fattispecie elusiva, pur derivando storicamente dal negozio in frode alla legge, presenta elementi di differenziazione dagli istituti dell'evasione, della simulazione e dell'abuso del diritto.

Difatti, l'evasione si ravvisa allorquando il contribuente occulta proventi o deduce costi inesistenti. A differenza del comportamento elusivo, l'evasione costituisce un vero e proprio illecito, che può integrare gli estremi di un'infrazione amministrativa, che implica l'applicazione di sanzioni tributarie amministrative, ovvero, nelle ipotesi più gravi, quelli di un reato penale, che comporta l'irrogazione di sanzioni tributarie penali. 
La simulazione integra ipotesi nelle quali le parti pongono in essere un regolamento soltanto apparente della fattispecie, mentre gli interessi funzionali al negozio sono in concreto inesistenti (simulazione assoluta) o diversi da quelli effettivamente perseguiti (simulazione relativa).

Dal punto di vista tributario, la simulazione assume rilievo allorquando è utilizzato un prestanome, che appare formalmente soggetto passivo d'imposta, nella cui sfera giuridica si produrranno gli effetti dell'atto e a carico del quale si applicherà il tributo; oppure nell'ipotesi in cui viene mascherata una certa operazione, attraverso l'attribuzione di una qualificazione giuridica che comporta l'applicazione di un trattamento fiscale meno oneroso.

Nell'«abuso del diritto», infine, il diritto soggettivo cessa di ricevere tutela, in quanto esercitato in difformità dai suoi limiti funzionali: il comportamento del titolare del diritto, malgrado la conformità formale alla disposizione normativa, tradisce le finalità intrinseche della norma.

Per quanto l'elusione fiscale prenda le mosse dal negozio in frode alla legge (art. 1344 cod. civ.), quindi, le due fattispecie presentano molteplici elementi discretivi: l'imperatività della norma tributaria riveste peculiarità diverse dall'imperatività dell'art. 1344 cod. civ., che tende ad impedire che i soggetti dell'ordinamento possano dare surrettizia attuazione a regolamenti negoziali in contrasto con interessi meritevoli di tutela.

In altri termini, l'art. 1344 cod. civ. si riferisce alle norme imperative di diritto privato, le quali, nell'individuare i limiti all'autonomia negoziale, inibiscono comportamenti diretti a raggirare un'autoregolamentazione dei rapporti privati difforme da quanto astrattamente previsto dal legislatore.

Questa connotazione finalistica esclude che la disposizione possa essere applicata ai negozi in frode al fisco (c.d. negotia in fraudem fisci). 


\section{OBEJŚCIE PRAWA JAKO SAMODZIELNA KATEGORIA PRAWA PODATKOWEGO}

Przypadek obejścia prawa i jest rozpowszechnionym zjawiskiem w systemie prawnym, obecnym w różnych dziedzinach prawa pozytywnego: w prawie cywilnym; w prawie administracyjnym; w prawie podatkowym. W tym ostatnim segmencie prawa fenomen jest znany jako "unikanie podatków".

W odróżnieniu od innych zjawisk, obchodzenie przepisów nie stanowi formalnej kategorii, ponieważ brakuje w nim elementu prawnego: chodzi w rzeczywistości o kategorię logiczna, zdolną do określenia założeń związanych z ominięciem zasad podatkowych.

Obejście przepisu podatkowego znaczy uniknięcie go poprzez doprowadzenie do odpowiednich okoliczności cywilnych lub poprzez działania w celu wyzerowania lub zmniejszenia obciążenia podatkowego.

Innymi słowami, obejście prawa dokonuje się więc, kiedy środki użyte do skonstruowania okoliczności z formalnego punktu widzenia prawa pozytywnego nie sprzeciwiają się przepisom prawa; w przeciwnym razie miałoby się do czynienia ze zjawiskiem unikania podatków.

Zjawisko obchodzenia prawa ma bardzo stare pochodzenie: już w źródłach prawa rzymskiego można znaleźć ślady tej instytucji. Obchodzenie prawa może być sprowadzone do starożytnego agere in fraudem legis, czyli zachowania ludzkiego, które, pomimo poszanowania poszczególnych przepisów prawnych, naruszało główne zasady prawodawstwa lub zasady pochodzące ze zbioru norm prawnych.

Obecnie termin «obejście prawa» określa sytuacje kiedy, pomimo poszanowania dla treści przepisu, jest łamana ratio legis, czyli duch, który wyraża przepis, z jednoczesną szkodą dla interesu chronionych.

W rzeczywistości, zjawisko obejścia prawa, pomimo, że historycznie pochodzi od aktu oszustwa, posiada elementy, które odróżniają je od instytucji unikania podatków, symulacji i nadużycia prawa.

W istocie, uznaje się za unikanie podatków sytuację, kiedy podatnik ukrywa przychody lub odejmuje od przychodu nieistniejące koszty. W odróżnieniu od działań dążących do obejścia prawa, unikanie opodatkowania stanowi prawdziwy i właściwy czyn niedozwolony, noszący znamiona wykroczenia administracyjnego, co wiąże się z nałożeniem kary grzywny za wykroczenie skarbowe, a w najbardziej 
skrajnych przypadkach, można mówić o przestępstwie karnym, za które grozi mandat karny.

Z symulacją mamy do czynienia, kiedy strony podejmują tylko pozorne działania, podczas gdy w rzeczywistości nie mają one wywołać skutków prawnych (symulacja absolutna) lub różnią się one od tych, do których dąży się w rzeczywistości (symulacja relatywna).

Z punktu widzenia prawa skarbowego symulacja jest istotna, kiedy zostaje użyty figurant, który będzie formalnie podatnikiem, dla którego będą wiążące efekty prawne aktu i który zostanie obciążony podatkiem; lub przypadek, kiedy zostaje ukartowana pewna operacja, której nadaje się taką kwalifikację prawną, która skutkuje mniejszym obciążeniem podatkowym.

Podczas «nadużycia prawa» prawo podmiotowe przestaje być chronione, ponieważ jest wykonywane w sposób sprzeczny z jego funkcjonalnymi ograniczeniami: zachowanie osoby uprawnionej, pomimo zgodności formalnej $z$ rozporządzeniem prawnym, zdradza jego prawdziwy cel.

Chociaż obejście podatków ma swoje początki wakcie oszustwa prawnego (art. 1344 cod. civ.), obie czynności posiadają wiele elementów, które je rozróżniają: imperatywność przepisu podatkowego nabiera specyfiki odmiennej od imperatywności art. 1344 cod. civ., który dąży do zakazania podmiotom możliwości podstępnego korzystania z przepisów prawnych w sposób sprzeczny z interesem publicznym.

Innymi słowy, art. 1344 cod. civ. odnosi się do imperatywnych zasad prawa prywatnego, które przy określaniu autonomii prawnej zabraniają czynów dążących do obejścia samoregulacji stosunków prywatnych różnej od tej przewidzianej w teorii przez ustawodawcę.

Ta celowa konotacja wyklucza możliwość zastosowania rozporządzenia do aktów oszustwa podatkowego ( negotia in fraudem fisci). 


\section{EVASION OF LAW AS THE CATEGORY OF TAX LAW}

The phenomenon of evasion is widespread in the legal system and present in different areas of positive law: in civil law; in administrative law; in tax law. In the latter, the phenomenon is known as "tax avoidance".

In contrast to other phenomena, the evasion of law is not a formal category, because it lacks the element of law: it is in fact a logical category, capable of determining the assumptions associated with the evasion of tax principles.

Evasion of the tax provision means its avoidance by implementing measures to reset or reduce the tax burden.

In other words, evasion of law occurs when the means used to construct the circumstances from the formal point of view of positive law is not in conflict with the provisions of the law; otherwise, one would have to deal with the phenomenon of avoiding taxes.

Key words: taxes, application of law, evasion, fraud 